IR Thermography for ASDEX Upgrade

\title{
Real Time Capable IR Thermography for ASDEX Upgrade
}

\author{
B.Sieglin, ${ }^{1, \text { a) }}$ M.Faitsch, ${ }^{1}$ A.Herrmann, ${ }^{1}$ B.Brucker, ${ }^{1}$ T.Eich, ${ }^{1}$ L.Kammerloher, ${ }^{1}$ and \\ S.Martinov ${ }^{1}$ \\ Max-Planck Institute for Plasma Physics, Boltzmannstr. 2, \\ D-85748 Garching
}

(Dated: 29 October 2015)

Infrared (IR) thermography is widely used in fusion research to study power exhaust and incident heat load onto the plasma facing components (PFC). Due to the short pulse duration of today's fusion experiments, IR systems have mostly been designed for off-line data analysis. For future long pulse devices (e.g. Wendelstein 7-X, ITER) a real time evaluation of the target temperature and heat flux is mandatory. This paper shows the development of a real time capable IR system for ASDEX Upgrade. A compact IR camera has been designed incorporating the necessary magnetic and electric shielding for the detector, cooler assembly. The camera communication is based on the Camera Link industry standard. The data acquisition hardware is based on National Instruments hardware, consisting of a PXIe chassis inside and a fibre optical connected industry computer outside the torus hall. Image processing and data evaluation is performed using real time LabVIEW.

a) Electronic mail: Bernhard.Sieglin@ipp.mpg.de 


\section{INTRODUCTION}

Infrared (IR) thermography is widely used in fusion research to study power exhaust and incident heat load onto the plasma facing components ${ }^{1-4}$. Most of the systems used today are commercially available IR cameras which are not specifically designed for the use on fusion experiments. The close proximity of the detector to the experiments leads to the exposure to strong magnetic fields and occasionally high voltage spikes. A number of commercially available cameras include electronics for image processing which has been left out in the camera design shown here and the processing is instead performed by the systems computer outside the torus hall. By removing all unnecessary electronics from the camera and by integrating the magnetic shielding into the camera design the volume and weight of the system is reduced drastically.

As interface with the detector the Camera Link standard ${ }^{5}$ is used. The data acquisition is based on National Instruments hardware consisting of a PXIe chassis inside and an industry computer outside the torus hall. Both components are connected via fibre optics providing the mandatory galvanic decoupling. Acquisition, image processing and data evaluation are implemented on real time LabVIEW using $\mathrm{C}$ for time critical and computational demanding tasks.

\section{IR CAMERA}

The IR camera developed for the use at ASDEX Upgrade contains the IR detector, an interface electronics board and the magnetic and electric shielding required for the operation in an environment with a high magnetic field. The IR detector used for the IR system is a digital 640x512 $15 \mu \mathrm{m}$ pitch InSb detector with an integrated Stirling cooler developed by $\mathrm{SCD}^{6}$. The detector has a spectral response from $\lambda=3.6-4.9 \mu \mathrm{m}$ limited by spectral filters. The resolution can be changed between 13-15 bit, with the higher resolution leading to a reduced frame rate. In full frame mode a frame rate of $300 \mathrm{~Hz}$ with 13 bit is possible. For a sub window frame rates of up to $5.5 \mathrm{kHz}$ can be achieved. In addition to the integration time and the resolution of the detector, the pixel capacity can be changed. Two settings are possible, one with the full integrator capacity of around $1 \mathrm{pA}$ and one with about a quarter of the full integrator capacity. This feature allows the adjustment of the sensitivity of the 
camera without the need to change the integration time. So far if low temperatures were to be measured a long integration time was needed, which reduced the achievable frame rate. Now the integrator capacity can be reduced increasing the achievable frame rate for the system. This extensive set of controls allows for a flexible setup of the camera based on the field of application. Figure 1 shows an illustration of the detector in the camera housing.

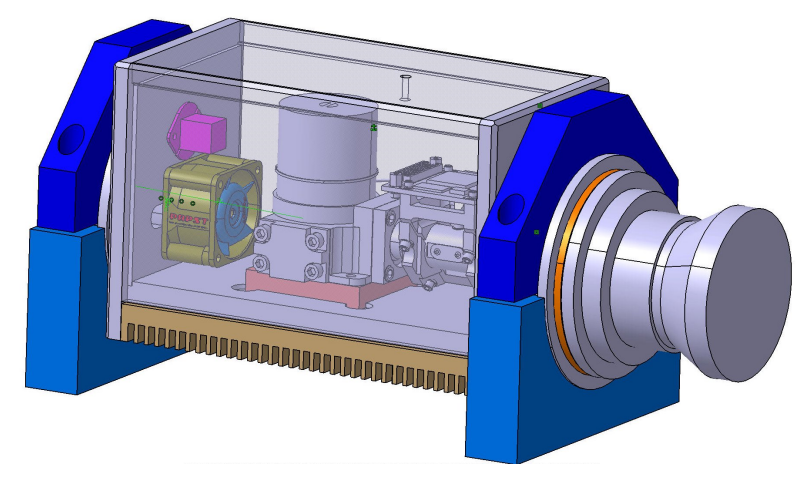

FIG. 1. Illustration of the detector in the camera housing (transparent) with rotatable mounting (blue).

The housing itself consists of $5 \mathrm{~mm}$ thick soft iron to provide magnetic shielding for the detector, the electronics and the Stirling cooler up to a field strength of around $100 \mathrm{mT}$. At ASDEX Upgrade the cameras are mounted outside the toroidal field coils where this shielding is sufficient. Mounted to the housing are two cylindrical parts at the front and the back of the camera. Together with the external mounting (blue in figure 1) these parts form a bearing allowing for the rotation of the camera around the optical axis. The option to rotate the camera is needed because the detector has a fast and a slow readout direction, with the fastest frame rate being obtained by using a narrow sub window of 640x4 pixels. The bearing allows the alignment of this narrow window to the measurement area, e.g. a poloidal line on the divertor target. The cylinders are manufactured from PEEK to provide electrical isolation of the camera from the mounting.

\section{ACQUISITION SYSTEM}

The IR detector itself is equipped with a proxy board which allows the communication with the detector using the Camera Link standard. An interface board providing the power supply and two SDR26 Camera Link connectors was designed. With this it is possible to 
use off the shelf cables to connect the camera to the data acquisition system.

For the communication with the camera and the data acquisition a system based on National Instruments hardware is used. During the operation of ASDEX Upgrade it is required to have no galvanic connection between the inside and the outside of the torus hall. To ensure this a fibre optic connection between the hardware in the torus hall and the computer on the outside was chosen, using National Instruments MXI-Express. This solution forwards the PCIe bus of the computer to the PXIe chassis using a 10GBase-CX4 active optical cable.

In the torus hall a PXIe chassis from National Instruments is used. The chassis is equipped with the camera link frame grabber card providing the communication with the camera and the acquisition of the frames during the discharge. In addition the power supply for the camera and an timing card for the synchronization with the ASDEX Upgrade discharge control system (DCS) is installed.

The computer operates with real time LabVIEW enabling the communication with the hardware in the PXIe chassis. Data acquisition and all time critical parts of the real time evaluation are implemented in C. The configuration, start and stop of the acquisition are implemented using LabVIEW.

\section{DATA ACQUISITION}

During the acquisition the system has to complete a variety of tasks. The most important task is the reading of the data from the camera and the consecutive saving of it. With data rates of up to 250 MBytes per second and a pulse duration of about 10 seconds for ASDEX Upgrade it would still be feasible to acquire the data to the main memory of the computer. With long pulse devices such as Wendelstein $7-\mathrm{X}$ in mind a solution was chosen where the data is streamed to a fast Solid State Disk (SSD). For a pulse duration of 30 minutes the data volume of around 440 GBytes is well within the scope of commercially available SSD hard drives.

For the acquisition and data evaluation itself a ring buffer is allocated in the main memory of the computer. The ring buffer contains the allocated memory for $N$ frames to be acquired. When a frame is acquired the process will point to the next memory block in the ring buffer. By doing this the previous frame is not overwritten when the next frame is acquired. When 
the end of the ring buffer is reached the whole process starts again at the first element of the buffer.

The system is designed in a way that existing and possible new camera types can be included in the acquisition software. Some cameras need data post processing (e.g. byte swapping, value inversion, deinterlacing), therefore a second ring buffer is allocated to provide memory for a RAW corrected image. A third ring buffer to hold the final corrected / calibrated image is allocated in addition.

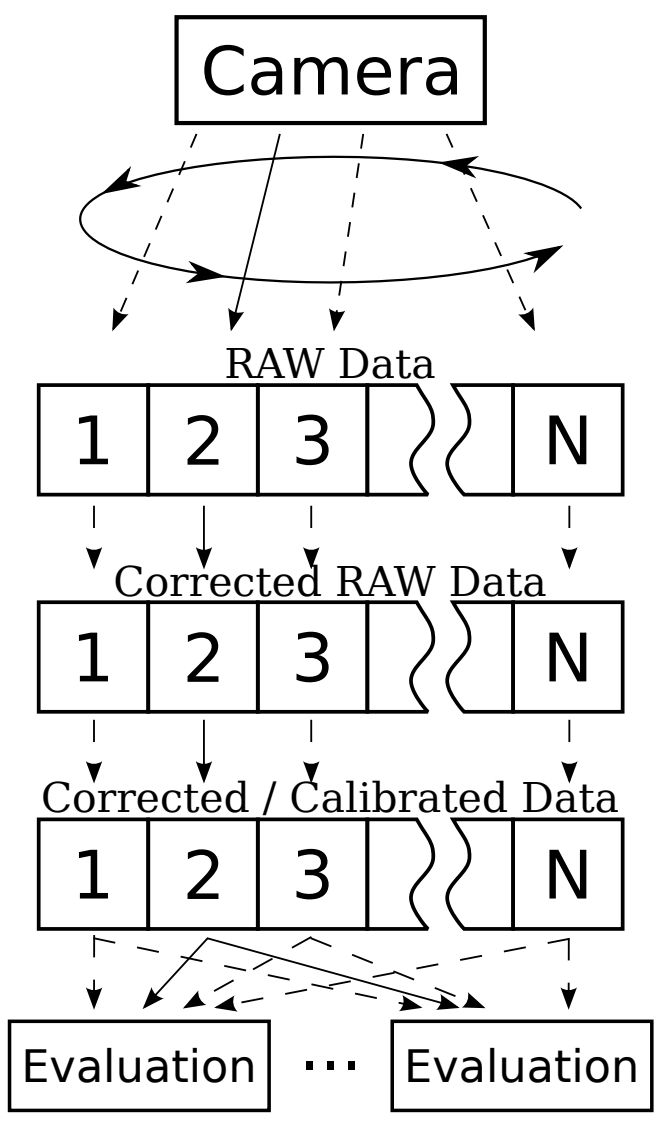

FIG. 2. Illustration of the ring buffers used for data acquisition and evaluation. $\mathrm{N}$ is the number of preallocated frames in the ring buffer.

A schematic representation of the evaluation work flow is shown in figure 2. After the frame is acquired the corresponding correction function is triggered to process the acquired ring buffer element. All processing steps and evaluations are done in separate threads in order not to block the acquisition of frames.

Once the post processing is completed the function for the correction / calibration of the image is invoked. This step can include gain and offset correction, replacement of corrupted 
pixels from the frame, temperature calibration and so on. A detailed description of the procedure used for the IR data is given in section $\mathrm{V}$.

Having finished the correction of the data the functions for the data evaluation are invoked. Multiple evaluation functions can be registered when the acquisition is configured. Examples for evaluation functions are given in section VI.

The complexity of the correction and evaluation functions is limited by the cycle time of the image acquisition. The computer needs the be able to complete the computations in the time between two frames. The real time system ensures that all tasks are scheduled accordingly and have priority before e.g. system tasks to ensure an unblocked execution of the acquisition. The system has been successfully commissioned for full frame and windowed mode up to the maximum frame rate, which results in a cycle time of $3.3 \mathrm{~ms}$ and around $180 \mu$ s respectively.

\section{IMAGE PROCESSING AND CALIBRATION}

The IR detector used in the camera uses a InSb CMOS sensor. Using CMOS has the advantage of a variable sub window read out as well as high frame rates compared to CCD sensors. Since every pixel on the detector has its own read out logic the response varies between pixels resulting in a non uniform image.

Before quantities like temperature or heat flux can be deduced from the acquired data, the RAW data has to be processed.

\section{A. Gain and Offset}

Before the discharge the detector gain and bias voltage are set to deliver a linear response for the expected range of the photon flux. Having set the detector settings a correction for the gain and offset has to be obtained to be able to calculate a uniform image. The read out electronics require to perform a separate gain offset correction for every combination of window size, integrator capacity, diode bias and resolution used in the experiment. The gain and offset correction is independent on the integration time of the system.

The most commonly used method is to measure a black body radiator at two different temperatures and then calculate the offset and gain. This method has a few disadvantages. 
Cavity radiators usually have only a small opening making it difficult to have a uniform illumination of the detector. Large area black body sources are an option if available. In the case of ASDEX Upgrade the use of a black body source is not practical (possible) to measure a black body when the camera is mounted at the machine.

The method proposed for the use in the experiment is based on an integration time series. The camera view is blocked using a shutter (e.g. lens cover). With the shutter in place an integration time series is acquired. In contrast to visible cameras the IR camera detects radiation coming from the shutter. The illumination is more or less homogeneous since the shutter is not in the focus plane. In this setup the photon flux reaching one pixel is constant. The expected digital level is proportional to the integration time.

With this the offset and the gain of each pixel are calculated. In order not to change the detector calibration it is advised to normalize the gain. Applying the correction to a RAW image results in a uniform image.

On ASDEX Upgrade this method proved to be useful. A shutter at room temperature provides a sufficiently high photon flux for the gain and offset correction. The acquisition of the integration time series requires less than one second and therefore no temperature control of the shutter is required. This method allows the in-situ measurement of the detector response by the use of a shutter in front of the camera.

\section{B. Bad Pixel Correction}

After the gain and offset correction the image can still contain pixels which deviate from the expected response. The value of such a bad pixel could be stuck at a low (dead) or high (hot) value. In addition pixels can have the expected response only in an interval of the digital level, in the following those pixels will also be accounted as bad pixels.

The bad pixels of a detector can be obtained by analyzing the gain and offset correction. Pixels that are stuck at a fixed value have no gain and can easily be recognized. For pixels with a deviating response a threshold for the deviation from the expected response can be set.

For the correction itself the value of the bad pixel is replaced according to the value of the neighbouring pixels. The exact replacement formula can be chosen in such a way that it gives the best quality for the application. For the operation at ASDEX Upgrade the pixel 
value is replaced with the mean average of its four nearest neighbours. In case of larger clusters of dead pixels this method is not suitable and different replacement schemes need to be tested.

\section{Temperature}

The correction so far delivers a uniform image without electronic readout background. From this image the surface temperature of the observed object is determined using Planck's law.

$$
\Gamma(\lambda, T)=\iint \epsilon \frac{2 \pi c}{\lambda^{4}} \frac{1}{\exp \left(\frac{h c}{\lambda k T}\right)-1} \mathrm{~d} A \mathrm{~d} \lambda
$$

Where $\Gamma$ is the photon flux, $\epsilon$ the emissivity of the surface, $\lambda$ the wavelength of the photons, $T$ the temperature of the surface, $h$ is Planck's constant, $c$ the velocity of light and $k$ is the Boltzmann constant.

Using filters to reduce the observed wavelength interval and assuming a homogeneous surface Eq. 1 is simplified.

$$
\Gamma(\lambda, T)=c_{0} \epsilon \frac{2 \pi c}{\lambda^{4}} \frac{1}{\exp \left(\frac{h c}{\lambda k T}\right)-1}
$$

$\lambda$ now denotes the effective wavelength of the filter used for the measurement. The constant $c_{0}$ contains the information about the solid angle and spectral bandwidth of the measurement. $c_{0}$ is obtained by measuring the photon flux of a black body reference source with known temperature and emissivity.

If the emissivity is independent of the temperature Eq. 2 can be solved for the surface temperature $T$.

$$
T=\frac{h c}{\lambda k \ln \left(\frac{2 \pi c c_{0} \epsilon}{\lambda^{4} \Gamma}+1\right)}
$$

For the real time system this function is used to calculate the surface temperature. In case of a temperature dependent emissivity, where Eq. 3 is not able to describe the calibration curve (Figure 3), an implementation using interpolation or a lookup table is possible.

The temperature calibration has to be done for each combination of lens, filter and detector. Windows and mirrors in the optical path have to be included in the calibration.

Using two different lenses (25 mm and a 1:1 makro lens) the calibration is performed to show the need for a separate calibration. For these measurements a band-pass filter 


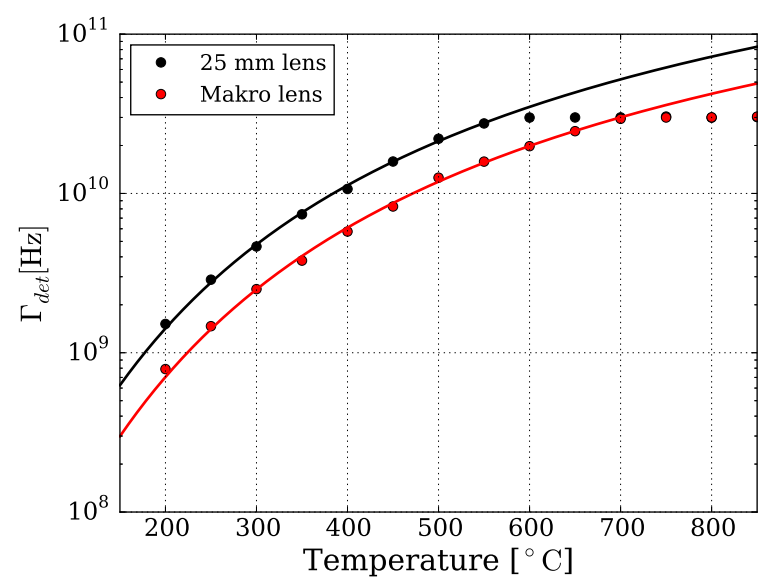

FIG. 3. Count rate in dependence of the temperature of the black body reference source. The measurement for the $25 \mathrm{~mm}$ lens are shown as black, the macro lens is shown as red dots. The fit of Eq. 3 to the data is shown as solid lines.

from $3.5-4.5 \mu \mathrm{m}$ is used. Figure 3 shows the measured count rate in dependence of the temperature of the black body reference. The $25 \mathrm{~mm}$ lens (black) has a higher count rate at the same temperature compared to the makro lens (red). The fit of Eq. 3 to the data is shown as solid line. It is seen that the model is able to describe the measured data, despite the large spectral bandwidth of the filter. At high photons fluxes the measurement saturates. This is due to the detector settings. At a count rate of $30 \mathrm{GHz}$ and the lowest used integration time of $1 \mu$ s the detector measures $3 \cdot 10^{5}$ events. Including the black current of the detector the 15 bit used for this measurement are reached, resulting in saturation.

The resulting maximum temperature that the system with this configuration is able to measure with an emissivity of 0.95 is $550{ }^{\circ} \mathrm{C}$ for the $25 \mathrm{~mm}$ lens and $650{ }^{\circ} \mathrm{C}$ for the makro lens. For a tungsten surface with a lower emissivity the maximum temperature is higher due to the lower photon flux. This has to be taken into account when choosing the lens, filter combination for the measurement.

\section{EVALUATION}

The real time system is developed with different applications in mind. Depending on the application different evaluation functions can be set for the acquisition. The main focus of the MWIR camera is the measurement of the heat flux distribution onto the plasma facing 
components in ASDEX Upgrade (e.g. First Wall, Divertor). It is possible to use different cameras, e.g. near infra red (NIR) cameras for machine protection or visible cameras for live view of the discharge, as long as they comply with the CameraLink standard. Only the setup prior to the discharge has to be implemented for each camera type, the acquisition itself is independent on the camera type.

\section{A. Heat Flux}

The system is able to calculate the surface temperature distribution in real time. To calculate the heat flux density a 1D line profile is extracted from the measured image using bilinear interpolation. The heat flux density is then obtained by consecutively solving the heat diffusion equation for the bulk of the tile (2D).

$$
\rho c_{p} \frac{\partial T}{\partial t}=\nabla(\kappa \nabla T)
$$

Where $\rho$ is the density, $c_{p}$ the specific heat capacity and $\kappa$ the heat conductivity of the target material. For this a real time version of the THEODOR ${ }^{7}$ code is used which is implemented in C. Like the standard version of THEODOR the code itself uses the following substitution.

$$
u(T)=\int_{0}^{T} \kappa\left(T^{\prime}\right) \mathrm{d} T^{\prime}
$$

Using this substitution the partial derivatives translate as follows

$$
\begin{gathered}
\frac{\partial u}{\partial t}=\frac{\partial u}{\partial T} \frac{\partial T}{\partial t}=\kappa(T) \frac{\partial T}{\partial t} \\
\frac{\partial u}{\partial x}=\frac{\partial u}{\partial T} \frac{\partial T}{\partial x}=\kappa(T) \frac{\partial T}{\partial x} \\
\frac{\partial^{2} u}{\partial x^{2}}=\frac{\partial}{\partial x}\left(\kappa(T) \frac{\partial T}{\partial x}\right)
\end{gathered}
$$

Where Eq. 6 is associated with the left side of Eq. 4 and Eq. 8 is the right hand side of Eq. 4. With this the non linear diffusion Eq. 4 simplifies to the linear diffusion equation.

$$
\frac{\partial u}{\partial t}=\frac{\kappa}{\rho c_{p}} \Delta u=D \Delta u
$$

In contrast to Eq. 4 this equation is linear because no operator acts on $\kappa$. Eq. 9 is solved using the explicit Euler method. In the code the material properties $\kappa(T)$ and $D(T)$ are 
described by the following analytic function.

$$
f(T)=a+\frac{b}{\left(1+\frac{T}{T_{0}}\right)^{2}}
$$

This function is able to describe the temperature dependence of the material properties and the resulting heat potential $u(T)$ is invertible analytically. This is necessary to calculate the diffusion coefficient $D(T)$ for the next time step after the diffusion equation has been solved.

After the heat diffusion equation is solved for one time step the heat flux density $q$ through the top of the tile is calculated using Fourier's law.

$$
q=-\kappa \nabla T=-\nabla u
$$

In the code the three top most grid points are used to calculate the gradient using the forward finite difference scheme.

$$
\begin{aligned}
q & =-\frac{\partial u}{\partial x} \\
& \approx \frac{3 u(x)-4 u(x+\delta x)+u(x+2 \delta x)}{2 \delta x}
\end{aligned}
$$

Where $\delta x$ is the resolution of the grid in the direction perpendicular to the tile surface.

\section{B. Adaptive Integration Time}

One of the main issues for IR thermography in present day devices is the high dynamic range of the expected photon flux from the measurement area. The temperature of the target can change from room temperature to over $1500 \mathrm{~K}$ within one plasma discharge in ASDEX Upgrade. So far the integration time had to be chosen, estimating the photon flux based on the plasma and heating parameters, before the discharge. This requires the experience of the person operating the camera and is a possible source of error.

The IR detector offers the possibility to change the integration time during acquisition with a resolution of $0.5 \mu \mathrm{s}$. The maximum integration time is limited by the frame rate of the acquisition, the minimum integration time is $0.5 \mu \mathrm{s}$. Once the desired integration time is sent, the detector will use it from the next acquired frame on. In principle it is possible to have a different integration time for every frame in the acquisition.

In the real time system an evaluation mode is implemented that changes the integration time based on the measured well fill of the detector. 
So far this method has been tested using the heating and cooling of a tungsten filament (Figure 4).

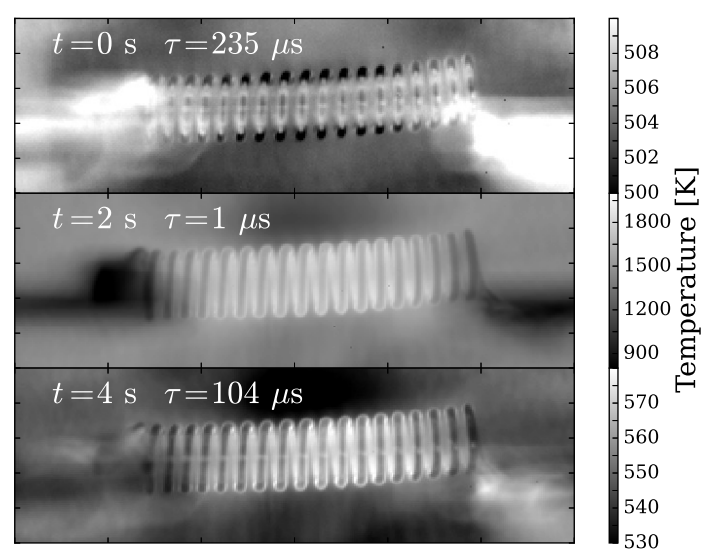

FIG. 4. Temperature of a tungsten filament before $(t=0 \mathrm{~s})$, during $(t=2 \mathrm{~s})$ and after $(t=4 \mathrm{~s})$ the heating pulse

To determine the well fill of the detector the calculation of a histogram is implemented. Histograms of frames before, during and after the heat pulse are shown in figure 5.

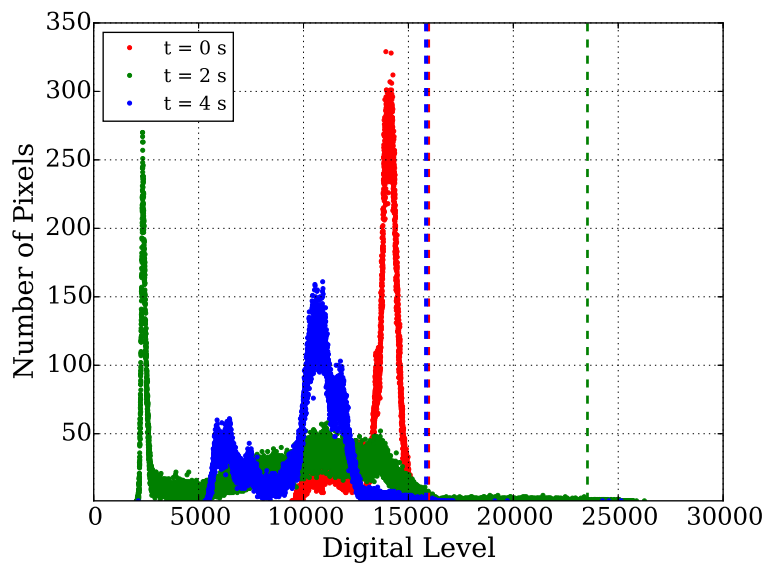

FIG. 5. Histogram of the frames before (red), during (green) and after (blue) the heat pulse. The 99.9 percentile is marked by the dashed lines.

In this experiment the adaptive integration time is set in order to keep the 99.9 percentile of the image at a value of 16000 , which is half of the dynamic range of the detector with 15 bit resolution. Before and after the heat pulse the percentile is close to the target value. Because the lower limit for the integration time was reached during the heat pulse the 
algorithm couldn't compensate, resulting in a higher value for the percentile.

A similar heating pulse has been acquired using a fixed integration time of $1 \mu$ s to compare the results to the acquisition with adaptive integration time. The resulting digital level of the measurement is shown in figure 6 .

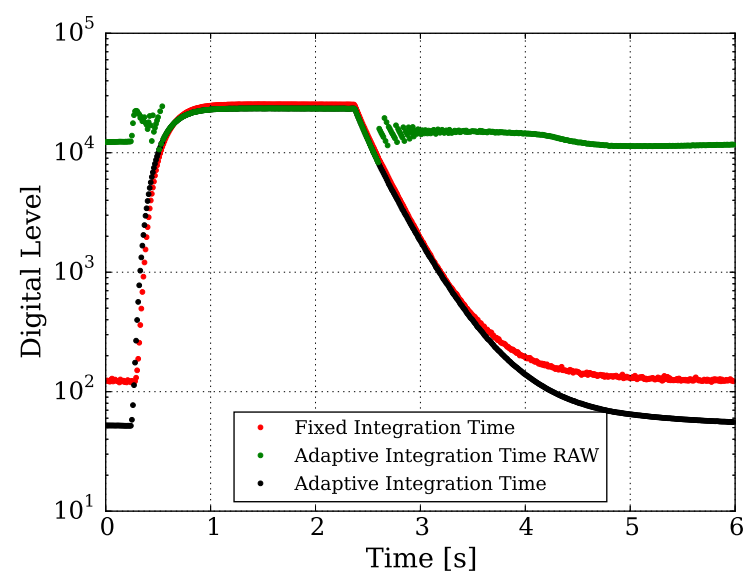

FIG. 6. Digital level of the measurement during the heating pulse with fixed integration time (red), with adaptive integration time (green) and the resulting reconstructed digital level (black).

The red curve shows the signal with the integration time fixed to $1 \mu \mathrm{s}$. The green curve is the signal with the adaptive integration time. It is seen that the system changes the integration time in order to get a high digital level without saturating the detector. The black curve shows the reconstructed signal obtained from the measurement with the adaptive integration time. The rise and fall of both measurements (red and black) are in agreement. Deviations are seen at low digital levels before $(t=0 \mathrm{~s})$ and after $(t=4 \mathrm{~s})$ the pulse. The signal with the adaptive integration time reaches a lower digital level compared to the signal with fixed integration time.

For the evaluation of the heat flux onto the plasma facing components at ASDEX Upgrade the noise level of the temperature measurement is crucial. The time derivative of the measured temperature is shown in figure 7. The heating (cooling) of the filament is seen as strong positive (negative) peak in the time derivative of the temperature. During the flat top of the heating pulse $(t=1-2 \mathrm{~s})$ the noise level of both signals is the same. This is to be expected since the digital level is similar. Before and after the heating pulse the noise level of both signals differ. The signal with adaptive integration time (black) has a significantly reduced noise level compared to the signal with fixed integration time (red). The 
reduced noise level is the consequence of the higher digital level of the signal with adaptive integration time compared to the one with constant integration time.

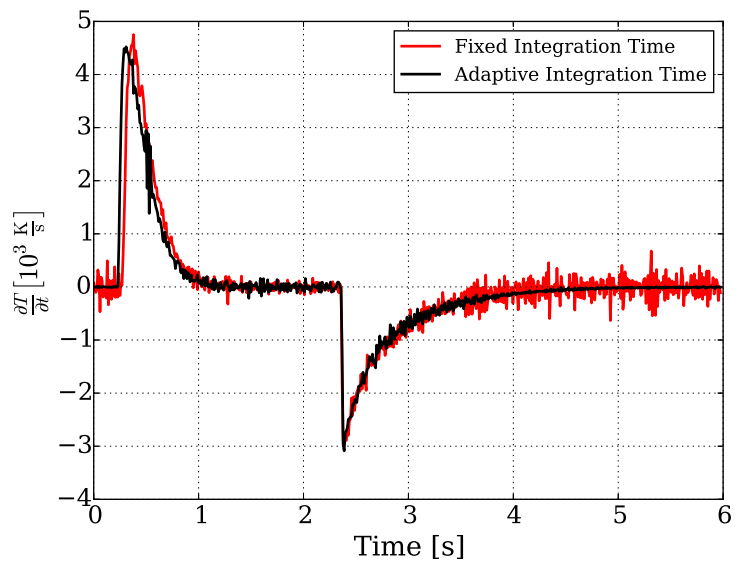

FIG. 7. Time derivative of the temperature during the pulse.

\section{CONCLUSIONS}

A real time capable IR system has been developed for ASDEX Upgrade. Using a digital detector by SCD a camera was designed and built which incorporates magnetic and electric shielding, reducing volume and weight. Real time data acquisition and evaluation based on commercially available hardware was implemented, allowing the use of different cameras based on the Camera Link standard. Long pulse acquisition ( $>30$ minutes) is possible due to the use of fast solid state disks. A variety of evaluation functions is implemented such as hot spot detection using ROIs or temperature profile evaluation. On-line heat flux evaluation is possible using a real time version of the heat flux evaluation code THEODOR. Exploiting the detectors feature to change the integration time during acquisition, the system is able to adapt the integration time based on the acquired image in real time, reducing the noise level of the measurement.

\section{ACKNOWLEDGEMENT}

This work has been carried out within the framework of the EUROfusion Consortium and has received funding from the Euratom research and training programme 2014-2018 under 
IR Thermography for ASDEX Upgrade

grant agreement No 633053. The views and opinions expressed herein do not necessarily reflect those of the European Commission.

\section{REFERENCES}

${ }^{1}$ D. Hildebrandt, F. Gadelmeier, P. Grigull, K. McCormick, D. Naujoks, D. Snder, and W7-AS Team. Thermographic observation of the divertor target plates in the stellarators w7-as and w7-x. Journal of Nuclear Materials, 313316(0):738 - 742, 2003. ISSN 0022-3115. doi:http://dx.doi.org/10.1016/S0022-3115(02)01490-3. URL http: //www . sciencedirect . com/science/article/pii/S0022311502014903. Plasma-Surface Interactions in Controlled Fusion Devices 15.

${ }^{2}$ E. Gauthier, H. Roche, E. Thomas, S. Droineau, B. Bertrand, J.B. Migozzi, W. Vliegenthart, L. Dague, P. Andrew, T. Tiscornia, and D. Sands. Iter-like wide-angle infrared thermography and visible observation diagnostic using reflective optics. $F u$ sion Engineering and Design, 82(514):1335 - 1340, 2007. ISSN 0920-3796. doi: http://dx.doi.org/10.1016/j.fusengdes.2007.06.026. URL http://www.sciencedirect . com/science/article/pii/S0920379607003158. Proceedings of the 24th Symposium on Fusion Technology SOFT-24.

${ }^{3}$ I. Balboa, G. Arnoux, T. Eich, B. Sieglin, S. Devaux, W. Zeidner, C. Morlock, U. Kruezi, G. Sergienko, D. Kinna, P. D. Thomas, M. Rack, and JET EFDA Contributors. Upgrade of the infrared camera diagnostics for the jet iter-like wall divertora). Review of Scientific Instruments, 83(10):10, 2012.

${ }^{4}$ Masaki TAKEUCHI, Tatsuo SUGIE, Hiroaki OGAWA, Masao ISHIKAWA, Takahiko SHIMADA, and Yoshinori KUSAMA. Development of divertor ir thermography for iter. Plasma and Fusion Research, 8:2402147-2402147, 2013. doi:10.1585/pfr.8.2402147.

${ }^{5}$ Automated Imaging Association (AIA). URL http://www.visiononline.org.

${ }^{6}$ T. Markovitz, I. Pivnik, Z. Calahorra, E. Ilan, I. Hirsh, E. Zeierman, M. Eylon, E. Kahanov, I. Kogan, N. Fishler, M. Brumer, and I. Lukomsky. Digital 640x512 / 15m insb detector for high frame rate, high sensitivity, and low power applications, 2011. URL http://dx . doi .org/10.1117/12.883345.

${ }^{7}$ A Herrmann, W Junker, K Gunther, S Bosch, M Kaufmann, J Neuhauser, G Pautasso, Th Richter, and R Schneider. Energy flux to the asdex-upgrade divertor plates determined 
IR Thermography for ASDEX Upgrade

by thermography and calorimetry. Plasma Physics and Controlled Fusion, 37(1):17, 1995. URL http://stacks.iop.org/0741-3335/37/i=1/a=002. 